\title{
Human metapneumovirus induces autophagy through JNK and MEK/ERK pathway
}

\section{Pan Zhang}

Children's Hospital of Chongqing Medical University

\section{Suhua Chen}

Children's Hospital of Chongqing Medical University

\section{Hui Yang}

Children's Hospital of Chongqing Medical University

\section{Bin Tan}

Children's Hospital of Chongqing Medical University

Yao Zhao ( $\nabla$ zhaoy@cqmu.edu.cn )

Chongqing Medical University Affiliated Children's Hospital https://orcid.org/0000-0003-4550-9436

\section{Research article}

Keywords: Human metapneumovirus; Autophagy; Pathway; Viral Replication

Posted Date: November 14th, 2019

DOI: https://doi.org/10.21203/rs.2.17332/v1

License: (c) (1) This work is licensed under a Creative Commons Attribution 4.0 International License. Read Full License 


\section{Abstract}

Background: Human metapneumovirus (hMPV) is a ubiquitous respiratory pathogen, especially in infants and young children. Virus-host interaction affects viral replication and host immune responses. Autophagy plays an important role in virus-host interaction. Airway epithelial cells serve as the first line in host to against respiratory virus infection. However, it is still unknown whether autophagy is activated in human metapneumovirus (hMPV) infected host cells. Methods: In this study, we demonstrated the occurrence of autophagy through some autophagic features including the conversion of GFP-RFP-LC3 plasmid, RFP-LC3 puncta and expression of autophagic-related gene. The pathways involved in autophagy were detected by Western Blot and verified by specific pathway inhibitors. The relationship between the replication of hMPV and autophagy was tested by Rapamycin, 3-MA, siRNA-LC3 and pathway inhibitors. We also used BALB/c mice to verified the autophagy and pathways in hMPV infection. Results: We found that the GFP-RFP-LC3 plasmid, RFP-LC3 puncta and expression of ATG5, ATG7, Becllin1, LC3 were increased in hMPV group in vitro. JNK and MEK/ERK signaling pathway are activated in hMPV induced-autophagy and we confirmed the two pathways with specific inhibitors SP600125 and PD98059. Furthermore, we found that rapamycin suppressed hMPV infection significantly, but reversed when treated with autophagy inhibitor 3-MA, siRNA-LC3 and pathway inhibitors. hMPV infected mice also induce autophagy through JNK and MEK/ERK pathways. Conclusion: Taken together, our results show strong evidence that autophagy involved in hMPV infection through JNK, MEK/ERK pathway which plays an antiviral role in the process of hMPV infection.

\section{Background}

Human metapneumovirus (hMPV), was first isolated in 2001 in Netherland, [1] which belongs to the paramyxoviral subfamily of the Paramyxoviridae. Global epidemiological surveys show that hMPV is prevalent worldwide and the most infected one is subtype A.[2-4] hMPV is the second pathogen that causes severe lower respiratory tract infections only next to respiratory syncytial virus (RSV). which clinical symptoms are similar to RSV, hMPV can also aggravate the infection together with severe acute respiratory syndrome (SARS), [5] RSV and other influenza virus.[6] Despite hMPV has been discovered nearly 18 years, most of the current research is mainly focused on epidemiology, its infection mechanism is poorly understood. So far, there are no safe and effective ways to reduce viral replication. As an intracellular reproductive pathogen, the interaction between virus and the host affects the viral infection process and the cellular homeostasis. Several studies have revealed that cellular autophagy actively participates in various pathogenic infections and plays a crucial role [7-9]. In this study, we focused on the interaction between hMPV and host cell, especially the role of autophagy in hMPV infection.

Autophagy represents critical for virus-host interaction.[10,11] Autophagy is a self-balancing and selfprotection mechanism in cell, coexisting with apoptosis and necrosis.[12] Autophagy, an vital immune response mechanism, can be used to resist viral and bacterial infections, and plays an important role in the treatment of disease.[7, 13] The interaction between autophagy and virus is diverse. Studies have shown that Epstein-Barr virus (EBV), Varicella-zoster virus (VZV) and Human immunodeficiency virus type 
1 (HIV-1) could induce autophagy, Hepatitis B virus (HBV) also induce autophagy through the X protein or activates Beclin1 expression. Infection of influenza $A$ virus in mouse embryonic fibroblasts lead to the accumulation of autophagosomes.[14-17] Contrary to above virus, Kaposi S sarcoma herpesvirus (KSHV) and human papillomavirus (HVS) inhibit autophagy by expressing vFLIP (viral FLICE inhibitory protein). [18] Autophagy-related pathway like AMP-activated protein kinase (AMPK), mitogen-activated protein kinase (MAPK), mechanistic target of rapamycin (mTOR) or endoplasmic reticulum (ER) stress, they all could be induced by viral infection and may trigger autophagy.[19-22] Virus infection inducing or inhibiting autophagy through different pathways depending on the type of virus.

Virus induced-autophagy which in turn affects virus infection in different ways. On the one hand, autophagy has antiviral effects which could degrade virus directly or participate in the progress of transporting virus from endosome to autophagic lysosome for degradation.[23, 24] On the other hand, Viruses have evolved various strategies to escape or even use autophagy to promote their own replication. $[25,26]$ Although autophagy acts as a non-specific reaction, different virus cause different autophagy reaction and even the same virus could cause different autophagy reaction when infected different cells, these all indicate that autophagy is virus specific and cell specific. [27, 28] Little know about the existence of intracellular autophagy during hMPV infection which requires us to explore.

In this study, we investigated that hMPV-infected cells could induce autophagy. We also demonstrated the JNK and MEK/ERK pathways were activated in this process in vivo and vitro. When 3-MA, siRNA and SP600125/PD98059 were used to inhibit autophagy the hMPV replication increased, this result indicated that autophagy has a protective role in viral infection. Our results show that JNK and MEK/ERK signaling pathway are critical for hMPV induced-autophagy and autophagy inhibit hMPV replication in this interaction which provide us a new antiviral option by targeting autophagy.

\section{Methods}

\section{Cell culture}

Human bronchial epithelial cells $₫ 16 \mathrm{HBE} \otimes$ were purchased from China Center for Type Culture Collection, was cultured with Dulbecco's Modified Eagle Medium (DMEM, Gibco, USA) in an incubator at $37^{\circ} \mathrm{C}$ containing $5 \% \mathrm{CO} 2$. Besides, the culture medium contains $10 \%$ fetal bovine serum (FBS, Gibco, USA) and $1 \%$ penicillin-streptomycin antibiotics (PSA, Hyclone, USA).

\section{Virus culture}

hMPV was successfully recovered from full-length cDNA clones of hMPV NL/1/00 by reverse genetics as described previously. Because the phenomenon of cytopathic (CPE) is difficult to observe, we used PCR to detect viral titers to replace the virus strength. hMPV was cultured with Vero E6 cells, when the cytopathic lesion reached $80 \%$, the cells-virus mixture were frozen-thaws to release the virus particles and centrifuged at 4000rpm with $10 \mathrm{~min}$, the virus particles were suspended in medium and maintained as stocks at $80{ }^{\circ} \mathrm{C}$. A standard curve was drawn by double dilution of the standard hMPV F gene plasmid to 
detect the absolute quantification of viruses. Experimental group of viral RNA was extracted by using viral RNA extraction kit (Magen, China) and reverse transcribed into cDNA (Takara, Japan) for detection. Primer sequences designed with the conserved region of hMPV F gene

Forward primer sequence: GAGCAATAGCACTCGGTGTTG;

Reverse primer sequence: TCACAAATCTTTCAGCTCTCTCAC;

Probe sequence: TTGCCAACACACGAACTCCATCCC.

The reaction program was $95^{\circ} \mathrm{C}$ for $30 \mathrm{~s}, 40$ cycles of $95^{\circ} \mathrm{C}$ for $5 \mathrm{~s}$ and $60^{\circ} \mathrm{C}$ for $30 \mathrm{~s}$.

\section{Antibodies and reagents}

The following primary antibodies were used: rabbit anti-LC3 (L7543), rabbit anti-GAPDH (5174T), rabbit anti-SAPK/ JNK (9252T), rabbit anti-p SAPK/JNK (4668T), rabbit anti-ERK1/2 (4695T), rabbit anti-p ERK1/2 (437 0T), the following secondary antibody horse radish peroxidase (HRP)-conjugated goat anti rabbit (7074P2), all these antibodies were purchased from Cell Signaling Technology. The mouse antimetapneumovirus (ab 94804) is purchased from Abcam. The following regents: rapamycin (Rap, R0395), 3-methyladenine (3-MA, M9281), SP600125 (S5567) were purchased from Sigma-Aldrich. PD98059 (167869-21-8) was obtained from Selleck.

\section{Immunofluorescent LC3 plasmid}

LC3 is an important marker of autophagy, LC3II can accumulate on the membranes of autophagy precursors, autophagosomes and autophagy lysosome, so examining LC3II puncta can reflect the number of intracellular autophagic vesicles to assess the level of autophagy. Green fluorescence is easily degraded after autophagosome and lysosome fusion, but red fluorescence will not be disappeared, so double fluorescently labeled plasmid GFP-RFP-LC3II can directly reflect autophagy activity and autophagy flux. When autophagy was induced, both green and red fluorescence increased. The GFP-RFP-LC3 plasmid was given by BI Yang teacher. Plasmid was transfected with Lipofectamine 2000 (lip2000, 11668-027, Invitrogen) for 24h and observed under the fluorescence microscope. The control group was changed with medium (virus preservation solution) without hMPV, the virus treatment group was infected with hMPV and incubated in $37^{\circ} \mathrm{C}$. Confocal microscope was used to observe the GFP-RFP-LC3 plasmid fluorescence expression in $6 \mathrm{~h}$ and $24 \mathrm{~h}$ respectively.

\section{Small interference experiments.}

The following siRNA was designed:

siRNA-LC3 (sense, 5'-CUCCCUAAGAGGAUCUUUATT-3'; antisense, 5'-UAAAGAUCCUCUUAGGGAGTT-3'), the siRNA was designed by the Gene Pharma Company (Suzhou, China) and used to silence the expression of LC3 gene in 16HBE cells. The cells were transfected with different concentrations of siRNA using 
Lip2000 reagent according to the manufacturer's protocol and were harvested for further analysis after 24h, Western Blot was used to verify the silence efficiency of siRNA-LC3.

\section{RNA detection by PCR.}

We used RT-PCR to detect the expression of autophagy-related genes ATG5, ATG7, Beclin1 and the titer of hMPV. The same amount of $16 \mathrm{HBE}$ cells and the same weight of lung tissue were extracted from Ra Pure Total RNA Kit (Magen, R4011) according to the manufacturer. Prime Script TM RT reagent Kit (Takara, RR037A) was used to reverse the RNA into CDNA by following the procedures. We used SYBR (Qiagen, 216213) to semi-quantitatively detect the expression of related genes. The reaction program was $95^{\circ} \mathrm{C}$ for 2 min, 39 cycles of $95^{\circ} \mathrm{C}$ for $5 \mathrm{~s}$ and $60^{\circ} \mathrm{C}$ for $10 \mathrm{~s}$. The primer sequence of ATG5, ATG7, Beclin 1 were synthesized by Shanghai Shenggong Bioengineering Co, Ltd. These two reactions were conducted in a CFX96 Fluorescence Thermocycler (Bio-Rad, USA), and the data were analyzed with Bio-Rad CFX Manager software.

\section{Western blot}

Whole cell lysates were performed using RIPA lysis buffer (Beyotime, P0013B), 1 mM phenylmethanesulfonyl fluoride (PMSF, Beyotime, ST506-2) and protease phosphatase inhibitor mixture (Beyotime, P1045) in accordance with the manufacturer's protocol, which was performed on ice. Protein was extracted with the mixture and freeze at $-80^{\circ}$ for concentration determination. The protein concentration was measured using protein concentration assay kit (Beyotime, P0012S), protein denaturation using loading buffer 5X (Beyotime, P0015), SDS-gel configuration kit (Beyotime, P0012A). Protein loading per well is 30-60ug, after electrophoresis, the protein and other masses were transferred to a nitrocellulose membrane, blocked with $5 \%$ skim milk powder at $37^{\circ}$ for $1 \mathrm{~h}$, washed three times with TBST, and the antibody was diluted with $5 \%$ skim milk powder at $4^{\circ}, 60 \mathrm{rpm}$, overnight to incubate primary antibody. The secondary antibody was diluted with $2.5 \%$ skim milk powder, incubated at $37^{\circ}$ for $1 \mathrm{~h}$, washed three times with TSBS, and exposure with Millipore Immobilon ECL

\section{Confocal microscopy}

Cells were cultured on confocal four-plates. About plasmid transfection experiments, the plasmid GFPRFP-LC3 and lip2000 were diluted with serum-free medium at room temperature for $15 \mathrm{~min}$. Then transferred to cells for $6 \mathrm{~h}$ allow the plasmid entered cells, and replaced with fresh medium at $37^{\circ} \mathrm{C} 5 \%$ CO2. Stable expression of transfected cell lines infected with hMPV, then observe the fluorescence expression of the cells under confocal microscopy. For LC3 immunofluorescence staining, when the cells were fused to $60 \%-70 \%$, infected with hMPV for $4 \mathrm{~h}$, then cells were fixed in paraformaldehyde, and washed three times with PBS. Triton-X used to transparent (Sigma, Germany) for 15 min, $1 \%$ BSA blocked for 30 min, rabbit anti-LC3B Polyclonal Antibody (bs-2912R, Bioss) was incubated for $1 \mathrm{~h}$ at $37^{\circ}$, the second antibody was incubated for $1 \mathrm{~h}$, we added anti-fluorescence quencher to prevent fluorescence quenching and take a photograph with Nikon AIR confocal microscope. 


\section{Animal experiment.}

6-8 week old female BALB/c mice were purchased from the Experimental Animal Center of Chongqing Medical University and housed for three days prior to the experiment in a biosafety containment facility under the same conditions. Acclimated for three days and randomly divided into two groups, five in each group. BALB/c mice were anesthetized with $10 \%$ chloral hydrate, the airway of the mice was fully opened. The control group was given $10 \mu \mathrm{l}$ saline into both nostrils and the virus group treated with $10 \mu \mathrm{l} 10^{\wedge} 9$ hMPV virus. When the mice wheezed or coughed, patted the back and observed for 2-4 h. No abnormalities in the mice after waking up proved that the experimental treatment was successful. The mice were anesthetized by intraperitoneal injection with $10 \%$ chloral hydrate $(2.5 \mathrm{ml} / \mathrm{kg}$ body weight $)$ and sacrificed by cervical dislocation in five days after nasal drops, lung tissue was taken. $20 \mathrm{mg}$ of the left lung was used for protein extraction, added with RIPA (Biyuntian) and used Qiagen tissuelyser II to grind tissue with $25 \mathrm{HZ} / 10 \mathrm{~min}$. Take the supernatant after centrifugation, the supernatant was mixed with loading buffer and boiled, $-80^{\circ} \mathrm{C}$ preservation. $10 \mathrm{mg}$ of the left lung was used for RNA extraction, added with RNA extraction lysate and grind in the above manner, the supernatant was extracted according to the instructions (Magen single-package RNA extraction kit), and the Takara kit was used to reverse into cDNA, $-4{ }^{\circ} \mathrm{C}$ preservation. $10 \%$ formalin fixed the right upper lobe tissue for at least $24 \mathrm{~h}$. After fixation, put it into the embedding box and wash it overnight, embed it in paraffin, cut the tissue into $4 \mu \mathrm{m}$ thick slices, and observe the lungs pathological changes under the microscope after HE staining.

The experiment was repeated, and data were pooled.

\section{Statistical analysis}

Statistical evaluations were used the Student $t$ test, $P<0.05$ was considered statistically significant.

\section{Results}

\section{hMPV infection activates autophagy in 16HBE}

In order to explore the relationship between hMPV infection and autophagy, we first detected whether autophagy is triggered when hMPV infection. Immunofluorescence and the expression of autophagyrelated genes always be used to observe the existence of autophagy. We transfected the GFP-RFP-LC3II plasmid in 16HBE and set up for control group (only plasmid transfection), 6h-hMPV infection group and 24h-hMPV infection group (plasmid transfection+ hMPV infection). The result showed the green and red fluorescence spot was higher in $24 \mathrm{~h}$ infection group than in $6 \mathrm{~h}$, fluorescence intensity expression in a time-dependent after infection with hMPV (Fig. 1A, B). Then stain with RFP-LC3B antibody, result showed that LC3B was from diffuse to punctate distribution in hMPV group and had a significantly different to control group. We counted the number of intracellular LC3B spots in the control and hMPV groups (Fig. 1C). These data preliminary demonstrate that hMPV infection could induce autophagy in 16HBE. 
qPCR and western were used to detect the expression of autophagy-related genes. ATG5, Beclin1 and ATG7 were determined by SYBR Green and they all increased in hMPV infection group. (Fig. 1E). The autophagy-associated protein LC3II was increased and the expression was time-dependent, the highest expression of LC3II at $96 \mathrm{~h}$ (Fig. 1D). Taken together, these results confirmed the occurrence of autophagy after hMPV infection in 16HBE.

\section{hMPV infection induces the occurrence of autophagy through JNK and MEK/ERK pathway in 16HBE.}

Autophagy is a ubiquitous phenomenon in the process of cell survival, and there are many ways to induce autophagy. To clarify how hMPV infection induces autophagy, we first tested the mRNA expression of some autophagy-related pathways which were reported in previous article (This data is not displayed), finally we screened two genes which was constantly increased (Fig. 2A). Western Blot had the same results (Fig. 2B). These data suggested that the JNK pathway and the MEK/ERK pathway activated in hMPV-infected 16HBE.

To further confirm the involvement of JNK and MEK/ERK pathways in hMPV-induced autophagy, specific pathway inhibitor was used to make these two pathways ineffective during autophagy. 16HBE was treated with PD98059 (an inhibitor of the RAF/MEK/ERK pathway), and SP600125 (an inhibitor of the JNK pathway) before hMPV infection. (We used the CCK8 assay to observe drug-to-cell toxicity and found that different concentrations of the drug had no significant toxic effects on cells. The appropriate concentration was selected for subsequent experiments.) 16HBE was treated with 25 $\mu \mathrm{m}$ SP600125 and $10 \mu \mathrm{m}$ PD 98059, and then infected with hMPV, the expression of autophagy-related genes ATG5, ATG7, and Beclin1 decreased compared with control group (Fig. 2C). In addition, western blot showed the expression of autophagy-associated protein LC3-II was reduced after treated with inhibitors (Fig. 2D). Confocal microscopy was used to observe the RFP-LC3 spots in the hMPV and SP600125/PD98059 treatment groups. The autophagy spots in the virus group were obvious, but the spots in the drug group were decreased (Fig. 2E). Taken together, these results demonstrated that the pathway JNK and MEK/ERK does play a role in autophagy.

\section{Induction of autophagy regulates viral replication}

To confirm the role of autophagy in hMPV replication. We used Rapamycin (a universal autophagy inducer) and then checked its effect on viral load (Fig. 3A) We used western blot for hMPV protein and PCR for hMPV titer to represent viral load. High expressed autophagy reduced the replication of hMPV (Fig. 3B, C). 16HBE treated with 3-MA (a universal autophagy inhibitor) had an opposite result, the expression of LC3II decreased and hMPV replication raised (Fig.3A-C). These results indicate that autophagy displays an antiviral role in viral replication and that autophagy can inhibit the replication of the virus.

Silence autophagy by siRNA-LC3 and pathway inhibitor to verify the relationship between autophagy and viral replication 
In order to rule out the chemical effects of drugs, siRNA-LC3 was used to specific silence the LC3 gene in $16 \mathrm{HBE}$ to find the relationship between autophagy and viral replication. As showed in (Fig. 4A), 80 pmol siRNA-LC3 had an obvious inhibition. We built a siRNA-LC3 system and infected with hMPV (MOI=10), the virus titer was increased after infection compared with the blank-silencing group (MOI=10) (Fig. 4B). These results have showed that inhibit autophagy can promote viral replication.

The relationship between autophagy and hMPV replication was also clarified through specific pathway suppression. 16HBE was treated with the SP600125/PD98059and then infected with hMPV, the virus titer was significantly higher than that of the blank treatment group (Fig. 4D), viral protein expression was also increased (Fig. 4C). Taken together, it is clear that hMPV induces autophagy through the JNK and MEK/ERK pathways, and autophagy inhibits hMPV which plays a protective mechanism in $16 \mathrm{HBE}$.

\section{hMPV-induced autophagy occurs in mice through JNK, MEK/ERK pathway}

To verify whether hMPV-induced autophagy would occur in animals, BALB/c mice were used in this experiment. We divided the mice into control group and hMPV group $(n=5)$ by whether infected with or without hMPV. The mice in the control group were added with saline, hMPV group were added with $10^{\wedge} 7$ hMPV, then raised for 5 days under the same conditions. HE staining of mouse lung tissue showed thickening of the bronchial wall, inflammatory cell infiltration, and thickening of the alveolar septum (Fig. $5 \mathrm{E}$ ) in hMPV group. Compared with the control group, the autophagy-related gene expression was significantly higher in hMPV group (Fig. 5A). The autophagy-related protein was only expressed on the hMPV-infected group but not in the control group (The image only shows the strip of LC3II) (Fig. 5B). We further validated the expression of autophagy pathway JNK, MEK/ERK through RNA and protein level (Fig. 5C, D), and the results were consistent with cell experiments In general, we found that hMPV infection, like cells, induces autophagy via the JNK, MEK/ERK pathway in animals.

\section{Discussion}

The interaction between viral infection and autophagy is complex. Autophagy is essential in viral infection and play an important role on cellular homeostasis. Recent studies have demonstrated that pathogen infection could induce or inhibit autophagy. For example, Avian metapneumovirus (aMPV) and Japanese encephalitis virus (JEV) lead to the accumulation of autophagosomes in host cells, ER stress would involve in Hepatitis $\mathrm{C}$ virus (HCV) and Newcastle disease virus (NDV) induced autophagy while KSHV and HVS could inhibit autophagy. [19, 29-32] A large number of experimental studies have reported that cellular autophagy plays a role in Paramyxoviridae family members infected through different mechanisms in target cells.[33] However, there is no report showing whether hMPV induce autophagy and how autophagy affect hMPV replication. To elucidate this, we focused on the induction mechanisms of autophagy and the effect of autophagy on hMPV replication.

In the current study, transfection with GFP-RFP-LC3 plasmid showed increased fluorescent spots in hMPV infection group with time-dependent (Fig. 1A, B). Immunofluorescence showed the expression of LC3B 
spot in hMPV group was obvious (Fig. 1C). Furthermore, hMPV infection resulted in increased LC3II expression, and the mRNA expression of autophagy-related genes ATG5, ATG7, Beclin1 raised (Fig. 1D, E). Its worthy of note that the increasing in autophagy-gene expression and LC3 immunofluorescence could result from the occurrence of autophagy after hMPV infection. We discovered that hMPV infection induces autophagy in culture cell for the first time.

An increasing number of studies have reported that viruses could induce autophagy by activating diverse pathways. [34-36] Several studies have reported that ER stress after viral infection would lead to an unfolded protein responses in the endoplasmic reticulum, which could activate EIF2AK3, ATF6 or ERN1 pathway and induced the occurrence of autophagy.[19] Hepatitis $C$ virus induced autophagy via activated the EIF2AK3 (eukaryotic translation initiation factor 2 a kinase 3) and ATF6 UPR pathway or inhibit AKTTSC pathway,[37] [31] aMPV induces autophagy via the ATF6 pathway,[19] and PI3K/AKT/mTOR pathway,[38] Hepatitis B virus induces autophagy through PI3K/AKT/mTOR pathway,[39] Herpes simplex virus could down-regulated autophagy through homolog Bcl2.[40] Ras/PKA signaling pathway inhibits autophagy in Saccharomyces cerevisiae.[41] Autophagy is a complex physiological response mechanism, virus-induced autophagy pathway is virus specific. To explore the possible mechanism of hMPV induced-autophagy, we firstly screened a large quantity of pathway-related genes and found that the JNK and MEK/ERK pathways play an essential role in hMPV infected-cell (Fig. 2A, B). Although hMPV infection activated the JNK and MEK/ERK pathways, it is not certain that the above two pathways indeed exert effects in autophagy. Specific pathway inhibitors SP600125 and PD98059 were used to verify the accuracy of the two pathways. Results showed that the expression of autophagy-related genes were reduced after treat with inhibitors (Fig 2C, D). In this study, we analyzed the JNK and MEK/ERK pathways involved and play an important role in hMPV-induced autophagy. We also verified the existence of autophagy and JNK, MEK/ERK pathway played a role in hMPV-infected mice (Fig. 5). The two pathways are related to mitogen-activated protein kinase (MAPK) signaling pathway. MAPK can be activated by a series of extracellular signals, such as physical stress, inflammatory cytokines, viral bacteria, etc. The MEK/ERK pathway is a relatively clear pathway for current research, which is also involved in many pathophysiological processes such as cell growth and development, proliferation and differentiation. [42, 43] Extensive experimental researches have manifest that JNK signaling pathway have a crucial impact on cell differentiation, apoptosis, stress response and the occurrence and development of various diseases.[44] Therefore, JNK signaling pathway is an important regulatory target of cells in normal and disease states point.[44-46] Taken together, hMPV induces autophagy through the JNK and MEK/ERK pathways, which provides clues for understanding post-infection mechanisms and the search for therapeutic targets after infection. It is also convenient for us to understand the relationship between autophagy and viral replication.

The role of autophagy in virus replication is controversial, which mainly depends on the type of virus, the way of the virus infection and the type of cell and its microenvironment. $[47,48]$ During autophagy, virus, proteins and damaged organelles in the cytoplasm are encapsulated by autophagosomes with a bilayer membrane structure, and then through a series of membrane fusion processes, these substances are released into the lysosomes and then dissolve. Autophagy could directly degrade the virus or activated 
the immune response to exert antiviral effects. $[49,50]$ The usual host cell response is, virus promotes the accumulation of autophagy, which in turn inhibits the virus replication, a self-protective mechanism that cells develop as they grow. However, with the long-term virus escape immune mechanism, many viruses evolved to avoid the autocrine killing effect, and some viruses even use autophagy to promote their own replication. $[19,51]$ Zhou had reported that interference with autophagy impairs the accumulation of influenza A viruses M1 and M2 proteins.[52] Study indicated that hepatitis B virus (HBV) could induce autophagy to enhance its replication in cell cultures.[53] JEV replication could negative regulated by autophagy.[29] When autophagy was pharmacologically inhibited by wortmannin or LY294002, the replication of transmissible gastroenteritis virus (TGEV) increased and rapamycin treatment had the opposite conclusion. [54] In our study, the replication of hMPV decreased after the use of autophagy inducer-Rapamycin, while 3-MA had an opposite result.(Fig. 3A,C). In order to eliminate the influence of pharmacological reagent, we verified these results by silencing essential autophagy-related gene-LC3 (Fig 4A), hMPV replication was increased (Fig. 4B). In addition,SP600125 and PD98059 showed the viral replication was increased on RNA and protein level (Fig. 4C,D). In the present study, we found that inhibit autophagy may promote accumulation of viral RNA, improve viral titer and promote cell death. We have made a schematic diagram of intracellular autophagy after hMPV infection of 16HBE (Fig. 6). In conclusion, these results demonstrated the relationship between autophagy, JNK, MEK/ERK pathways and hMPV replication, our research supports the supposition that autophagy plays an antiviral role against hMPV infection which is a protective mechanism in 16HBE.

In previous studies, hMPV was cultured in Vero E6 cell which was the most suitable growth cells for hMPV. However, in this study, we performed experiments in 16HBE (human bronchial epithelial cells) to probe the respiratory response after hMPV infection. Airway epithelial cells serve as the first line of defense against hMPV infection, However, there is no study shows that the interaction of airway epithelial cells with autophagy after hMPV infection. 16HBE could better imitate the airway response of people with hMPV.

We have shown that JNK, MEK/ERK pathway involved in hMPV induced-autophagy. However, autophagy as a complex physiological mechanism in cell which pathway must more than above two. Therefore, the pathway of hMPV-induced autophagy still needs us to explore. In addition, it is not clear how hMPV triggered the above two pathways to induce autophagy. Which discussed more is ligand-receptor interaction, changes in intracellular lipid composition or intracellular homeostasis, stress response of endoplasmic reticulum. In the process of inducing autophagy, the formation of bilayer membrane enclosing virus may come from membrane organelles such as endoplasmic reticulum (ER) and golgi body. [55]When the homeostasis of ER is destroyed, a series of oxidative stress responses and immune responses will be induced, which may be closely related to the occurrence of autophagy. We have not conducted in-depth research, which requires follow-up exploration. This study showed that autophagy induced by hMPV infection in 16HBE could inhibit viral replication, but viral replication is not only associated with autophagy. Apoptosis, as a programmed death, is also important in viral infection and cell growth, which is closely related to viral replication. It has been reported that autophagy-mediated inhibition of apoptosis can promote RSV replication. Therefore, the physiological mechanism induced by 
hMPV infection needs further research and exploration. However, our research is also interesting. We have preliminarily demonstrated that autophagy can inhibit the intracellular replication of hMPV, which provides a new experimental basis for the research and treatment of hMPV infection.

In summary, we tested and verified that hMPV infection arouses autophagy for the first time, and this process could be regulated through JNK, MEK/ERK pathway. When we inhibited autophagy by siRNA-LC3 and pathway inhibitor, hMPV replication would reduce. These results further indicate that autophagy plays an antiviral respond and its positive protection during hMPV infection. Our study provides us a new insight into the process of hMPV infected host cells and discovery of new antiviral drugs or vaccine development by targeting autophagy.

\section{Conclusion}

We tested and verified that human metapneumovirus infection induce autophagy from cellular and animal experiments for the first time. The induction of autophagy is related to the activation of JNK and MEK/ERK pathways. The interaction between host cell and hMPV could be regulated by autophagy. Intracellular replication of the hMPV is associated with autophagy, viral replication is decreased when autophagy is promoted but increased when autophagy is inhibited. This experiment can help us find the targets to inhibit hMPV infection and control its replication, and provide a theoretical basis for the development of antiviral drugs and vaccines.

\section{Declarations}

\section{Ethics approval and consent to participate}

The Chongqing Science and Technology Commission approved the production and use of the Experimental Animal Center of Chongqing Medical University. The production license number is SCXK(Yu)2018-0003, and the license number is SYXK(Yu)2018-0003. Animal care and use according to the "Regulations on the Management of Laboratory Animals" of the Ministry of Science and Technology and the National Laboratory for Quality and Technical Supervision (GB14922-2001 to GBT14927-2001).

\section{Consent for publication}

Not applicable

\section{Availability of data and materials}

All data generated or analysed during this study are included in this published article [and its supplementary information files].

\section{Competing interests}

There is no conflict of interest in this study. 


\section{Funding}

This research was supported by National Natural Science Foundation of China (NO. 30800972, 81371876, 81701997), Natural Science Foundation Project of CQ and YuZhong District CSTC (NO. cstc2017jcyjAX0043 and 20170403), Chongqing Municipal Colleges and Universities Outstanding Talent Support Program (2014-47-111), Outstanding Youth Fund of Children's Hospital of Chongqing Medical University. No funding bodies had any role in the design of the study, collection, analysis, and interpretation of data and in writing the manuscript.

\section{Authors' Contributions}

$\mathrm{YZ}$ and $\mathrm{PZ}$ conceived and designed this study. $\mathrm{PZ}, \mathrm{SH}$ and $\mathrm{HY}$ performed the experiments. $\mathrm{PZ}, \mathrm{SH}, \mathrm{HY}$ and BT analyzed and interpreted the date. PZ wrote the manuscript. YZ supervised the project. Manuscript was proofread by $Y Z$. All authors approved the final version of the article, including the authorship list.

\section{Acknowledgements}

We thank Bi Yang for providing the GFP-RFP-LC3 plasmid in this study.

\section{References}

1. van den Hoogen BG, de Jong JC, Groen J, Kuiken T, de Groot R, Fouchier RA, Osterhaus AD: A newly discovered human pneumovirus isolated from young children with respiratory tract disease. Nat Med 2001, 7(6):719-724.

2. Apostoli P, Zicari S, Lo Presti A, Ciccozzi M, Ciotti M, Caruso A, Fiorentini S: Human metapneumovirus-associated hospital admissions over five consecutive epidemic seasons: evidence for alternating circulation of different genotypes. J Med Virol 2012, 84(3):511-516.

3. Banerjee S, Sullender WM, Choudekar A, John C, Tyagi V, Fowler K, Lefkowitz EJ, Broor S: Detection and genetic diversity of human metapneumovirus in hospitalized children with acute respiratory infections in India. J Med Virol 2011, 83(10):1799-1810.

4. Canducci F, Debiaggi M, Sampaolo M, Marinozzi MC, Berre S, Terulla C, Gargantini G, Cambieri P, Romero E, Clementi M: Two-year prospective study of single infections and co-infections by respiratory syncytial virus and viruses identified recently in infants with acute respiratory disease. $J$ Med Virol 2008, 80(4):716-723.

5. Lee N, Chan PK, Yu IT, Tsoi KK, Lui G, Sung JJ, Cockram CS: Co-circulation of human metapneumovirus and SARS-associated coronavirus during a major nosocomial SARS outbreak in Hong Kong. J Clin Virol 2007, 40(4):333-337.

6. Manoha C, Espinosa S, Aho SL, Huet F, Pothier P: Epidemiological and clinical features of hMPV, RSV and RVs infections in young children. J Clin Virol 2007, 38(3):221-226.

7. Levine B, Kroemer G: Autophagy in the pathogenesis of disease. Cell 2008, 132(1):27-42. 
8. Randow F, Munz C: Autophagy in the regulation of pathogen replication and adaptive immunity. Trends Immunol 2012, 33(10):475-487.

9. Colombo MI: Autophagy: a pathogen driven process. IUBMB Life 2007, 59(4-5):238-242.

10. Viret C, Rozieres A, Faure M: Autophagy during Early Virus-Host Cell Interactions. J Mol Bio/ 2018, 430(12):1696-1713.

11. Jordan TX, Randall G: Manipulation or capitulation: virus interactions with autophagy. Microbes Infect 2012, 14(2):126-139.

12. Yoshimori T: Autophagy: a regulated bulk degradation process inside cells. Biochem Biophys Res Commun 2004, 313(2):453-458.

13. Shintani T, Klionsky DJ: Autophagy in health and disease: a doubleedged sword. Science 2004, 306(5698):990-995.

14. Sir D, Tian YJ, Chen WL, Ann DK, Yen TSB, Ou JHJ: The early autophagic pathway is activated by hepatitis B virus and required for viral DNA replication. Proceedings of the National Academy of Sciences of the United States of America 2010, 107(9):4383-4388.

15. Lee DY, Sugden B: The LMP1 oncogene of EBV activates PERK and the unfolded protein response to drive its own synthesis. Blood 2008, 111(4):2280-2289.

16. Gannage M, Dormann D, Albrecht R, Dengjel J, Torossi T, Ramer PC, Lee M, Strowig T, Arrey F, Conenello $\mathrm{G}$ et al: Matrix protein 2 of influenza A virus blocks autophagosome fusion with lysosomes. Cell Host Microbe 2009, 6(4):367-380.

17. Zhou XB, Spangle JM, Munger K: Expression of a viral oncoprotein in normal human epithelial cells triggers an autophagy-related process. Autophagy 2009, 5(4):578-579.

18. Lee JS, Li QL, Lee JY, Lee SH, Jeong JH, Lee HR, Chang H, Zhou FC, Gao SJ, Liang CY et al: FLIPmediated autophagy regulation in cell death control. Nat Cell Bio/ 2009, 11(11):1355-U1225.

19. Hou L, Wei L, Zhu SS, Wang J, Quan R, Li ZX, Liu J: Avian metapneumovirus subgroup C induces autophagy through the ATF6 UPR pathway. Autophagy 2017, 13(10):1709-1721.

20. Zhou YY, Li Y, Jiang WQ, Zhou LF: MAPK/JNK signalling: a potential autophagy regulation pathway. Bioscience Rep 2015, 35.

21. Zhang Q, Zhu HX, Xu XS, Li LY, Tan HM, Cai XY: Inactivated Sendai virus induces apoptosis and autophagy via the PI3K/Akt/mTOR/p70S6K pathway in human non-small cell lung cancer cells. Biochem Bioph Res Co 2015, 465(1):64-70.

22. Mihaylova MM, Shaw RJ: The AMPK signalling pathway coordinates cell growth, autophagy and metabolism. Nat Cell Biol 2011, 13(9):1016-1023.

23. Kobayashi S, Orba Y, Yamaguchi H, Takahashi K, Sasaki M, Hasebe R, Kimura T, Sawa H: Autophagy inhibits viral genome replication and gene expression stages in West Nile virus infection. Virus Res 2014, 191:83-91.

24. Hafren A, Macia JL, Love AJ, Milner JJ, Drucker M, Hofius D: Selective autophagy limits cauliflower mosaic virus infection by NBR1-mediated targeting of viral capsid protein and particles. Proc Nat/ 
Acad Sci U S A 2017, 114(10):E2026-E2035.

25. Chiramel Al, Best SM: Role of autophagy in Zika virus infection and pathogenesis. Virus Res 2018, 254:34-40.

26. Richetta C, Gregoire IP, Verlhac P, Azocar O, Baguet J, Flacher M, Tangy F, Rabourdin-Combe C, Faure M: Sustained autophagy contributes to measles virus infectivity. PLoS Pathog 2013, 9(9):e1003599.

27. Paul P, Munz C: Autophagy and Mammalian Viruses: Roles in Immune Response, Viral Replication, and Beyond. Advances in Virus Research, Vol 952016, 95:149-195.

28. Sumpter R, Levine B: Selective autophagy and viruses. Autophagy 2011, 7(3):260-265.

29. Sharma M, Bhattacharyya S, Nain M, Kaur M, Sood V, Gupta V, Khasa R, Abdin MZ, Vrati S, Kalia M: Japanese encephalitis virus replication is negatively regulated by autophagy and occurs on LC3-Iand EDEM1-containing membranes. Autophagy 2014, 10(9):1637-1651.

30. Ma J, Sun Q, Mi R, Zhang H: Avian influenza A virus H5N1 causes autophagy-mediated cell death through suppression of mTOR signaling. J Genet Genomics 2011, 38(11):533-537.

31. Huang H, Kang RY, Wang J, Luo GX, Yang W, Zhao ZD: Hepatitis C virus inhibits AKT-tuberous sclerosis complex (TSC), the mechanistic target of rapamycin (MTOR) pathway, through endoplasmic reticulum stress to induce autophagy. Autophagy 2013, 9(2):175-195.

32. Meng C, Zhou Z, Jiang K, Yu S, Jia L, Wu Y, Liu Y, Meng S, Ding C: Newcastle disease virus triggers autophagy in U251 glioma cells to enhance virus replication. Arch Virol 2012, 157(6):1011-1018.

33. Joubert PE, Meiffren G, Gregoire IP, Pontini G, Richetta C, Flacher M, Azocar O, Vidalain PO, Vidal M, Lotteau V et al: Autophagy Induction by the Pathogen Receptor CD46. Cell Host \& Microbe 2009, 6(4):354-366.

34. Schuck S, Gallagher CM, Walter P: ER-phagy mediates selective degradation of endoplasmic reticulum independently of the core autophagy machinery. Journal of Cell Science 2014, 127(18):4078-4088.

35. He C, Klionsky DJ: Regulation mechanisms and signaling pathways of autophagy. Annu Rev Genet 2009, 43:67-93.

36. Fouillet A, Levet C, Virgone A, Robin M, Dourlen P, Rieusset J, Belaidi E, Ovize M, Touret M, Nataf S et al: ER stress inhibits neuronal death by promoting autophagy. Autophagy 2012, 8(6):915-926.

37. Wang J, Kang RY, Huang H, Xi XY, Wang B, Wang JW, Zhao ZD: Hepatitis C virus core protein activates autophagy through EIF2AK3 and ATF6 UPR pathway-mediated MAP1LC3B and ATG12 expression. Autophagy 2014, 10(5):766-784.

38. Zhou Y, Geng PP, Liu YL, Wu JY, Qiao HT, Xie YP, Yin N, Chen LL, Lin XC, Liu Y et al: Rotavirus-encoded virus-like small RNA triggers autophagy by targeting IGF1R via the PI3K/Akt/mTOR pathway. Biochimica Et Biophysica Acta-Molecular Basis of Disease 2018, 1864(1):60-68.

39. Mao Y, Da L, Tang H, Yang JL, Lei YR, Tiollais P, Li TP, Zhao MJ: Hepatitis B virus X protein reduces starvation-induced cell death through activation of autophagy and inhibition of mitochondrial apoptotic pathway. Biochem Bioph Res Co 2011, 415(1):68-74. 
40. Liang CG, Xiaofei E, Jung JU: Downregulation of autophagy by herpesvirus Bcl-2 homologs. Autophagy 2008, 4(3):268-272.

41. Cagnol S, Chambard JC: ERK and cell death: Mechanisms of ERK-induced cell death - apoptosis, autophagy and senescence. Febs Journal 2010, 277(1):2-21.

42. Boilly B, Vercoutter-Edouart AS, Hondermarck H, Nurcombe V, Le Bourhis X: FGF signals for cell proliferation and migration through different pathways. Cytokine \& Growth Factor Reviews 2000, 11(4):295-302.

43. Cagnol S, Chambard JC: ERK and cell death: mechanisms of ERK-induced cell death-apoptosis, autophagy and senescence. FEBS J 2010, 277(1):2-21.

44. Sui X, Kong N, Ye L, Han W, Zhou J, Zhang Q, He C, Pan H: p38 and JNK MAPK pathways control the balance of apoptosis and autophagy in response to chemotherapeutic agents. Cancer Lett 2014, 344(2):174-179.

45. Weston CR, Davis RJ: The JNK signal transduction pathway. Curr Opin Genet Dev 2002, 12(1):14-21.

46. Li L, He Q, Huang X, Man Y, Zhou Y, Wang S, Wang J, Li J: NOX3-derived reactive oxygen species promote TNF-alpha-induced reductions in hepatocyte glycogen levels via a JNK pathway. FEBS Lett 2010, 584(5):995-1000.

47. Jackson WT: Viruses and the autophagy pathway. Virology 2015, 479-480:450-456.

48. Wileman T: Aggresomes and autophagy generate sites for virus replication. Science 2006, 312(5775):875-878.

49. Orvedahl A, MacPherson S, Sumpter R, Jr., Talloczy Z, Zou Z, Levine B: Autophagy protects against Sindbis virus infection of the central nervous system. Cell Host Microbe 2010, 7(2):115-127.

50. Owczarczyk AB, Schaller MA, Reed M, Rasky AJ, Lombard DB, Lukacs NW: Sirtuin 1 Regulates Dendritic Cell Activation and Autophagy during Respiratory Syncytial Virus-Induced Immune Responses. J Immunol 2015, 195(4):1637-1646.

51. Sumpter R, Jr., Levine B: Selective autophagy and viruses. Autophagy 2011, 7(3):260-265.

52. Zhou Z, Jiang XJ, Liu D, Fan Z, Hu XD, Yan JG, Wang M, Gao GF: Autophagy is involved in influenza A virus replication. Autophagy 2009, 5(3):321-328.

53. Tian Y, Sir D, Kuo CF, Ann DK, Ou JH: Autophagy required for hepatitis B virus replication in transgenic mice. J Virol 2011, 85(24):13453-13456.

54. Guo L, Yu H, Gu W, Luo X, Li R, Zhang J, Xu Y, Yang L, Shen N, Feng L et al: Autophagy Negatively Regulates Transmissible Gastroenteritis Virus Replication. Sci Rep 2016, 6:23864.

55. Geng JF, Klionsky DJ: The Golgi as a potential membrane source for autophagy. Autophagy 2010, 6(7):950-951.

\section{Figures}




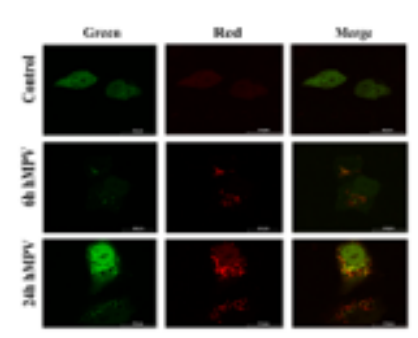

b

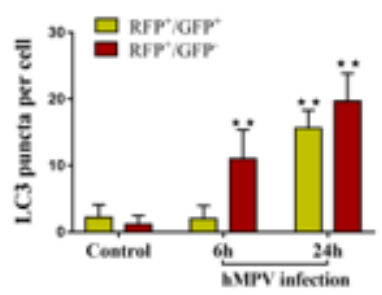

c
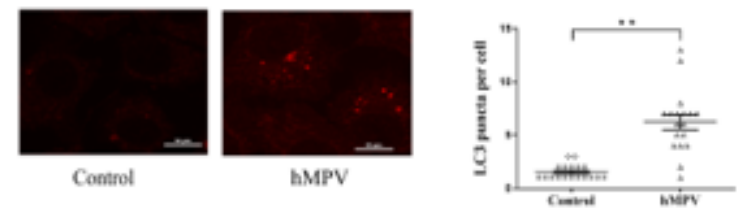

d

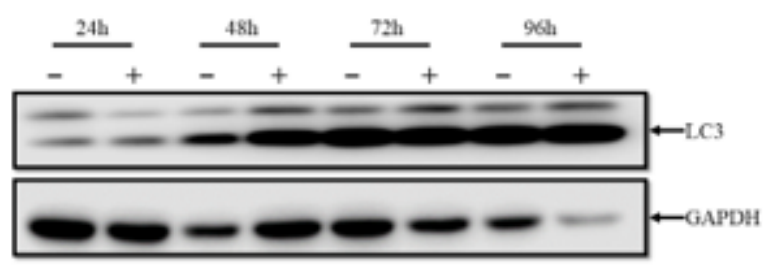

$\mathbf{e}$

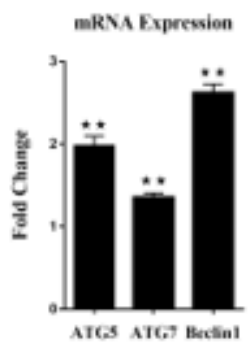

Figure 1

hMPV infection induces autophagy in 16HBE. (a) 16HBE was transfected with GFP-RFP-LC3 plasmid with Lip2000. GFP-RFP-LC3 expressing cells were divided into three groups: control group, infected with purified $\mathrm{hMPV}(\mathrm{MOl}=5)$ at $6 \mathrm{~h}$ and $24 \mathrm{~h}$ groups. The cells were then imaged by confocal microscopy. The bar was $20 \mu \mathrm{m}$. (b) The average LC3 spot in each cell was shown in the figure. (c) 16HBE were cultured in regular serum-containing medium or purified hMPV infected for 4h (MOI=5), LC3 spot expression was 
observed under confocal microscope, bar is $10 \mu \mathrm{m}$. Quantification of the number of LC3 puncta per cells. (d) Western blot detected LC3 in hMPV infected-group (MOI=10) or mock-group at the indicated times. The grouping of blots used for comparison were cropped from different parts of the same gel. (e) QPCR detected the expression of autophagy-related gene ATG5, ATG7, Beclin1 in mock infection group and hMPV infection group (MOI=10, 24h). The Student $t$ test was used to calculate $P$ values. $\mathrm{ALP}<0.01$.

a

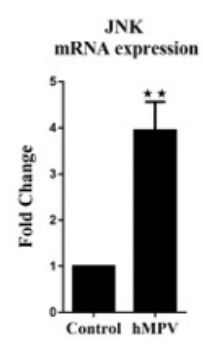

b
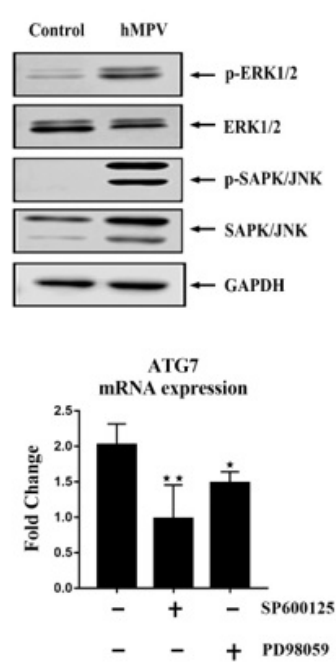

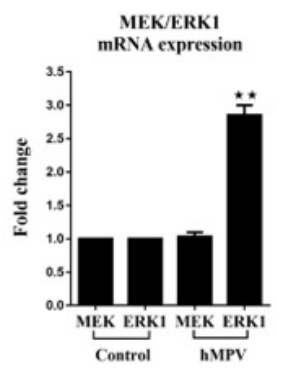

c
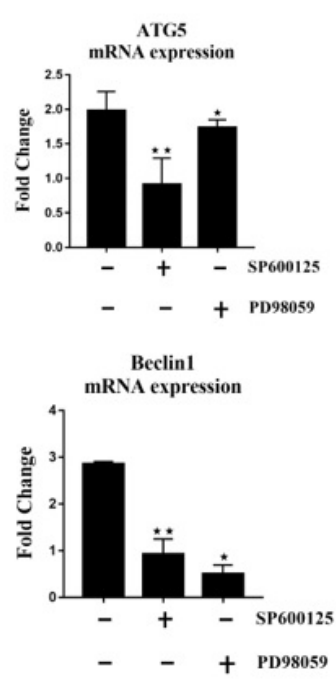

d
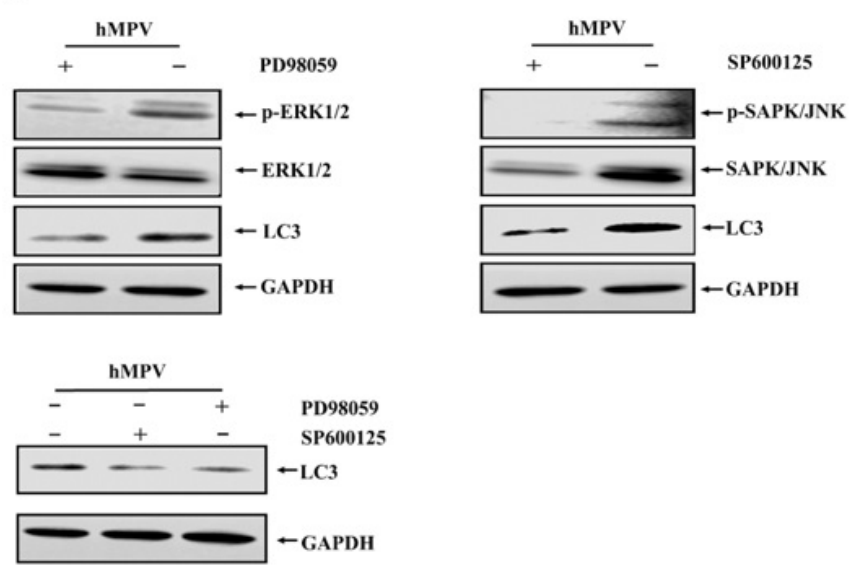

e
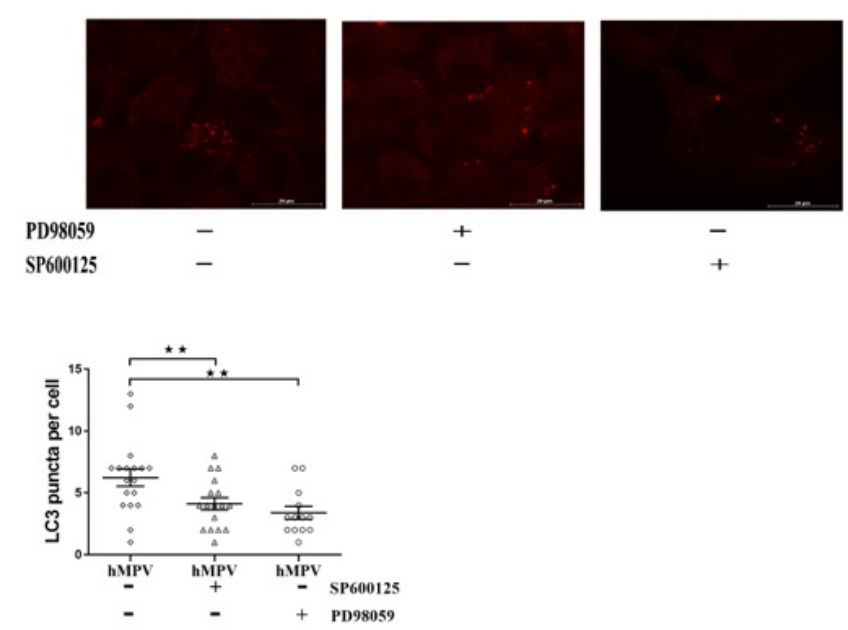

\section{Figure 2}

hMPV infection 16HBE induces the occurrence of autophagy through JNK and MEK/ERK pathway. (a) $16 \mathrm{HBE}$ cells were divided into mock infection group and $\mathrm{hMPV}$ infection group ( $\mathrm{MOI}=10,24 \mathrm{~h})$. QPCR to detect the pathway-related genes. (b) Western blot showed the expression of the JNK and ERK proteins and its phosphorylation form. The grouping of blots used for comparison were cropped from different parts of the same gel. (c) 16HBE were grouped into the following three groups: hMPV, hMPV+SP600125 $(25 \mu \mathrm{mol} / \mathrm{l}), \mathrm{hMPV}+\mathrm{PD} 98059(10 \mu \mathrm{mol} / \mathrm{l})$. The drug was pre-treated for 4 hours and then aspirated, infected with hMPV (MOI=10) for 24 hours to detect the expression of ATG5, ATG7, Beclin1 as shown in 
the figure. (d) Western blot indicated the inhibition of the two agents and detected the expression of LC3II. The blots were cropped, and the full-length blots are included in a Supplementary Information file (LC3 protein only shows LC3II after drug treatment). (e) The inhibitors were treated for $4 \mathrm{~h}$ and then infected with hMPV (MOI=5). The expression of LC3 was observed by confocal microscopy, the right panel shows the number of LC3 spots observed in each cell and statistical analysis indicates significant differences. The Student $t$ test was used to calculate $P$ values. $\square \mathrm{P}<0.05, \mathrm{QP} P<0.01$.

a

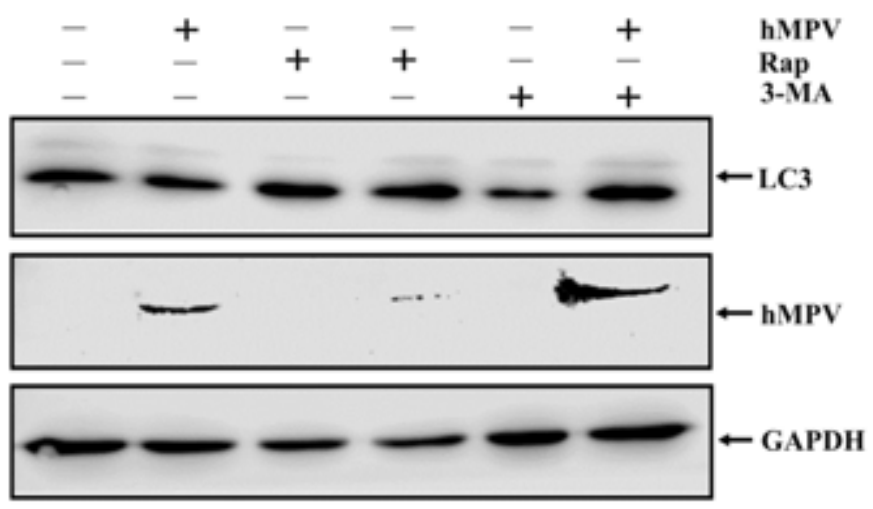

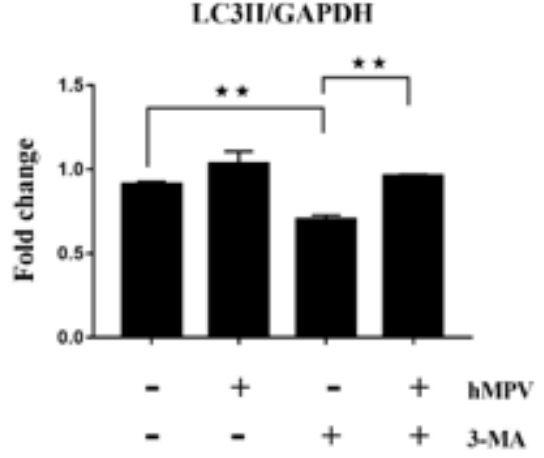

b

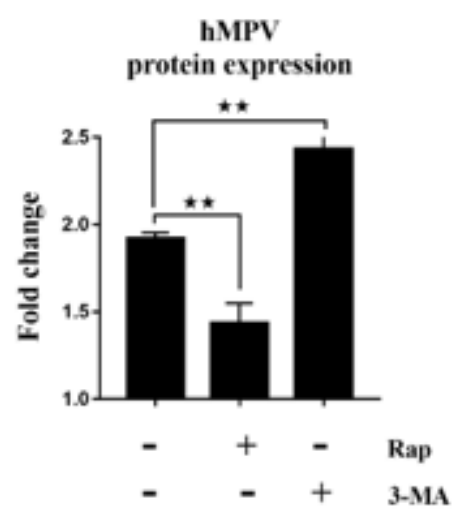

LC3II/GAPDH

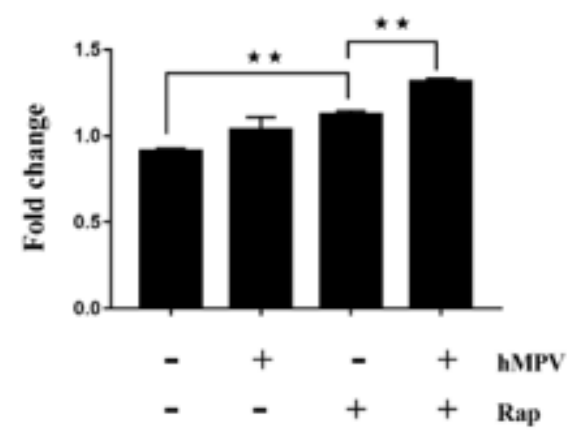

c

hMPV titer

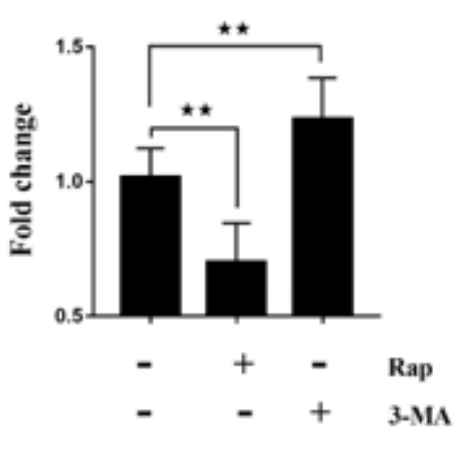

Figure 3 
Induction of autophagy regulates viral replication. 16HBE were pretreated or unpretreated with specific drugs Rapamycin $(10 \mu \mathrm{mol} / \mathrm{l})$ and $3 \mathrm{MA}(10 \mu \mathrm{mol} / \mathrm{l})$ for 4 hours, then washed and infected with hMPV (MOI=10). Infection with $16 \mathrm{HBE}$ for $48 \mathrm{~h}$, the protein expression of (a) LC3 and (b) hMPV were detected by western blot. The grouping of blots used for comparison were cropped from different parts of the same gel. (c) The same treatment but infection with 24h, RNA was used to detected hMPV titer by probe method. The Student $t$ test was used to calculate $P$ values. $\square P<0.05, \square \mathrm{P}<0.01$.

a

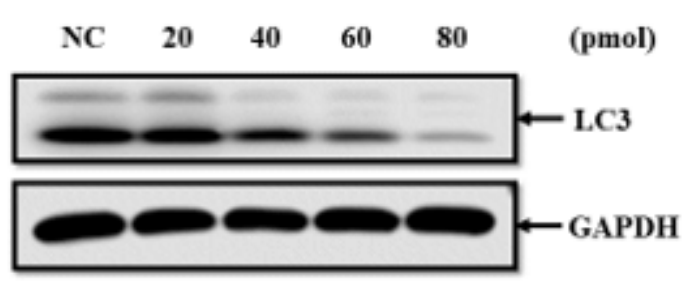

c

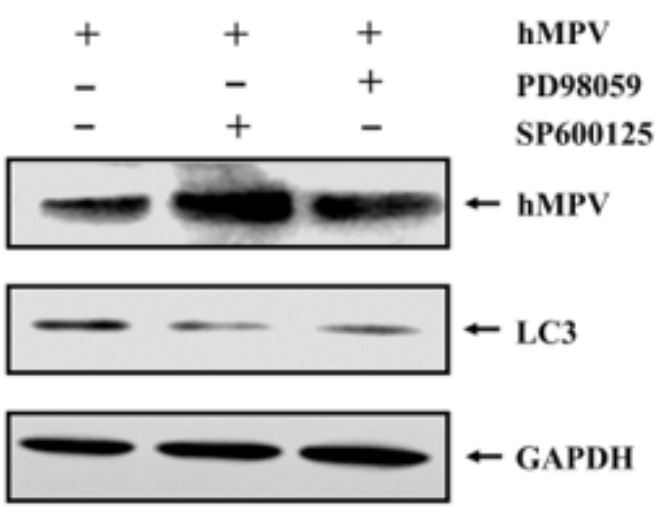

b

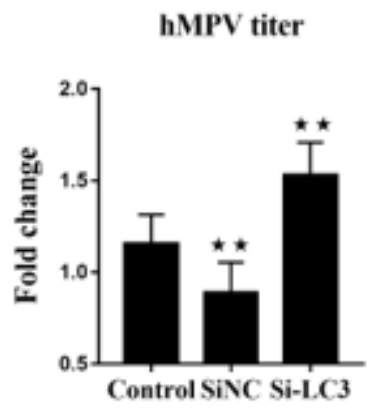

d

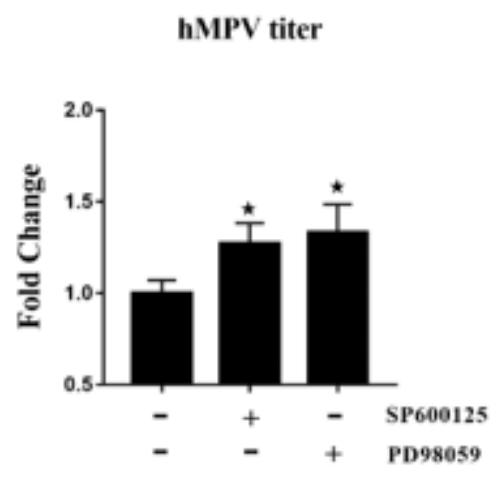

\section{Figure 4}

Silence autophagy by siRNA-LC3 and pathway inhibitor to verify the relationship between autophagy and viral replication. (a) siRNA was used to silence LC3 and, the gene silencing condition detected by western. There was a good silencing effect at $80 \mathrm{p} \mathrm{mol}$, and the concentration was used for subsequent experiments. The grouping of blots used for comparison were cropped from different parts of the same gel. (b) This concentration was used to construct the siRNA-LC3 silencing system, then infected with hMPV, virus titer was detected by PCR. (c) 16HBE were pretreated with specific pathway inhibitor drugs SP600125 $(25 \mu \mathrm{mol} / \mathrm{L})$ and PD98059 $(10 \mu \mathrm{mol} / \mathrm{L})$ for 4 hours, then washed and infected with virus (MOI=10, 48h), hMPV protein was detected by western blot. The blots were cropped, and the full-length blots are included in a Supplementary Information file. (d) hMPV titer was detected by QPCR after using SP600125 and PD98059. The Student $t$ test was used to calculate $P$ values. $\square P<0.05, \square \mathrm{P}<0.01$ Figure-5 Autophagy exists after hMPV infection in animals, and autophagy is induced by JNK and MEK/ERK pathways. 
$\mathbf{a}$

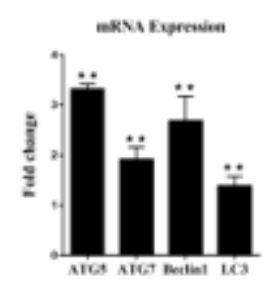

c

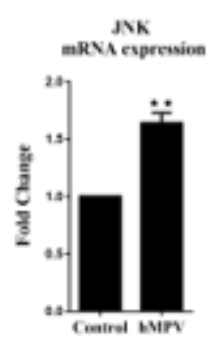

d

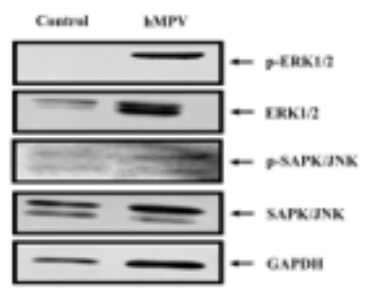

$\mathbf{e}$

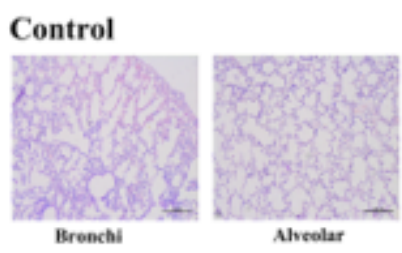

hMPV

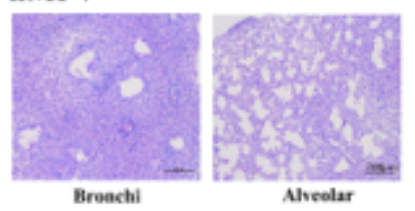

Figure 5

Autophagy exists after hMPV infection in animals, and autophagy is induced by JNK and MEK/ERK pathways. The animals were divided into the control group and the hMPV-infected group $(n=5)$. The hMPV-infected group nasally dropped with $10^{\wedge} 7$ virus, the control group was given equal capacity of saline nasal. The mice were sacrificed five days later to extract lung tissue RNA and protein. (a) RNA detected the expression of ATG5, ATG7, Beclin1, LC3. (b) Western blot detected the expression of LC3 
(LC3 protein only shows LC3II in mouse protein). (c and d) The pathway gene JNK, MEK/ERK expression test from RNA levels and protein levels were increased. The grouping of blots used for comparison were cropped from different parts of the same gel. (e) The lung bronchi and alveolar HE staining of mouse. The Student $t$ test was used to calculate $P$ values. $0 . P<0.01$.

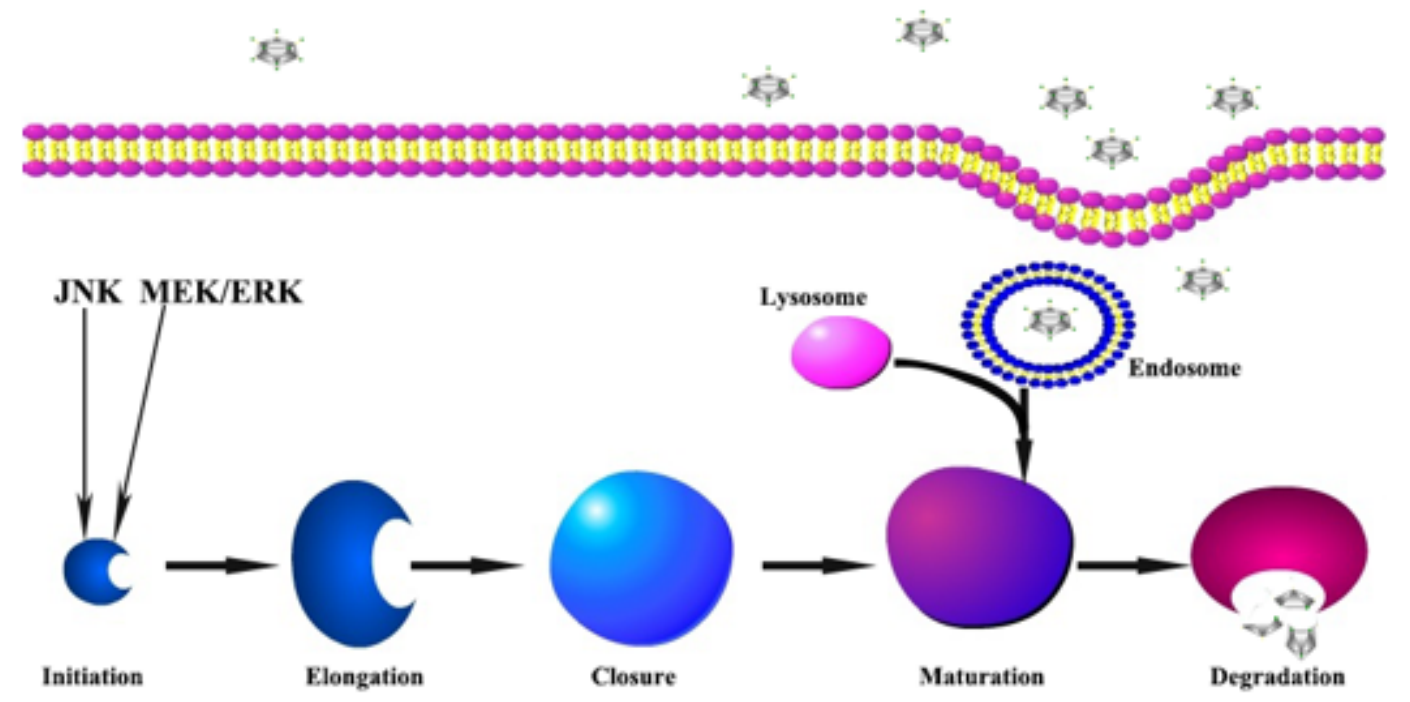

\section{Figure 6}

Schematic diagram of intracellular autophagy after hMPV infection of 16HBE. Our study found that hMPV induces autophagy through the JNK and MEK pathways, and this intracellular physiological response inhibits viral replication and protects cells.

\section{Supplementary Files}

This is a list of supplementary files associated with this preprint. Click to download.

- Supporting20191105.docx

- ARRIVEGuidelinesChecklist.pdf 